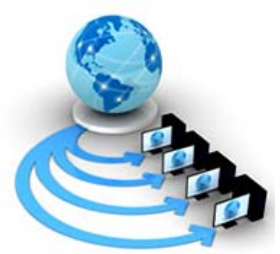

Volume 9, No. 1, January-February 2018

ISSN No. 0976-5697

International Journal of Advanced Research in Computer Science

REVIEW ARTICLE

\author{
Available Online at www.ijarcs.info
}

\title{
DEVELOPMENT OF COMPUTERIZED SYSTEMS FOR AUTOMATED CHROMOSOME ANALYSIS: CURRENT STATUS AND FUTURE PROSPECTS
}

\author{
Mousami V. Munot \\ Associate Professor, Dept. of E\&TC, \\ Pune Institute of Computer Technology, \\ Pune, India, 411043,
}

\begin{abstract}
Computer Aided Diagnosis (CAD) is an important pattern recognition application in the field of medical sciences. Such systems assist (not replaces) doctors in the interpretation of medical image. Computerized system for automated chromosome analysis is amongst popular CAD systems which have attracted the attention of numerous researchers, making it a thrust area for further investigations. In last 25 years, extensiveinvestigations have been contributed to designAutomated Karyotyping Systems (AKS). This paper presents a comprehensive survey of the developments and current trends in the field of AKS. The survey details the overall advancements in AKS since its origin and presents an effective review of pre-processing and image enhancement techniques, segmentation methods, feature extraction algorithms and the classifiers used in AKS. Few unaddressed issues and challenges that have comparatively received meagre attention are discussed highlighting the future prospects of AKS and providing pointers to the further research.
\end{abstract}

Keywords: Computer Aided Diagnosis, Pattern Recognition, Chromosomes, Karyotyping

\section{INTRODUCTION}

In cellular images, chromosomes are the constructions that contain hereditary information. Every cell in a normal human being has around $6 \times 10^{9}$ bits of information. These informative structures also called as information carriers of our body are located in the nucleus of the cell and are composed of genes that constitute the genome and genetic information of an individual. Genes control the physical characteristics of a species. Chromosomes are the key factors carrying valuable information regarding the health of an individual and therefore important to the doctors for detecting genetic syndromes [1]. Individuals belonging to a particular species or generic have exactly the same count of chromosomes in their nuclei [2]. A normal human being cell has 46 number chromosomes which are classified as belonging to either an autosomes category or sex /gender chromosomes. A standard human cell contains 22 pairs of autosomes and one pair of gender chromosomes, the $\mathrm{X}$ or Y. A female cell has a pair of X chromosome and having a combination of $\mathrm{X}$ and $\mathrm{Y}$ chromosome confirms the gender as male.

Process of cell cycle includes cell division as an important task. Normally, human chromosomes are particularly stretched and slender and are fundamentally invisible. However, during the metaphase stage of cell division, they become relatively much shorter and wider [1, 3]. During metaphase stage, chromosomes differ from each other in their morphology. The neck of the chromosome, centromere connects its shorter and the larger arm. The position of the centromere and the Centromeric Index (CI) which is also called as arm ratio (ratio between the lengths of the two chromosome arms) are the most prominent morphological features used for chromosome classification. Staining processes are used to separate chromosomes that are similar in size and centromere position [3].Ideogram is a standard representation for chromosomes which indicate it'srelative size and banding pattern $[4,5,6]$. These features are used in manual and automated karyotyping.

\section{KARYOTYPING}

In the process of reproduction, each parent (father and mother) contributes in formation of 22 homologous autosomes and a pair of gender chromosomes (23 pairs in a normal human being). A standard representation of these 23 pairs of chromosomes is known as a Karyogram. A normal karyogram has chromosomes belonging to three major groups (Metacentric, Submetacentric and Accrocentric) and seven sub-groups (A to G). Such a nomenclature is referred to as Denver Classification in the literature [7]. Karyotyping is the process of classifying the chromosomes in its respective groups or classes as per the ISCN standard (International Standard for Chromosome Nomenclature, 1997) [7] illustrates the karyogram of human cell based on Denver Group, detailing the subclasses, size and relative position of centromere for each group. Figure1 depicts a sample of metaphase image and its ordered karyotype from the publically available database [8]. The karyogram or the process of the karyotyping is the first fundamental step in identification of genetic disorders. It authenticates the exact count of the chromosomes in the cell which immediately confirms the possibility of up-syndrome or down-syndrome genetic disorders and thus provides the pointers to further diagnostic path.

\section{A. Manual Karyotyping:}

Traditional chromosome karyotyping is performed manually. A cytogenetic analyst uses high end microscope to capture an image during the metaphase stage of the cell division and identifies each chromosome in that image. The chromosomes are initially classified in the broader groups based on size and position of centromere. The next step of fine-detailing in 23 smaller groups needs expert knowledge 
and tremendous experience. The manual karyotyping methods are laborious, lengthy and tedious, dependent on trained cytogenetics and expert knowledge, time consuming, prone to human erroneous, have storage problem and are expensive, thus demanding automation [9].

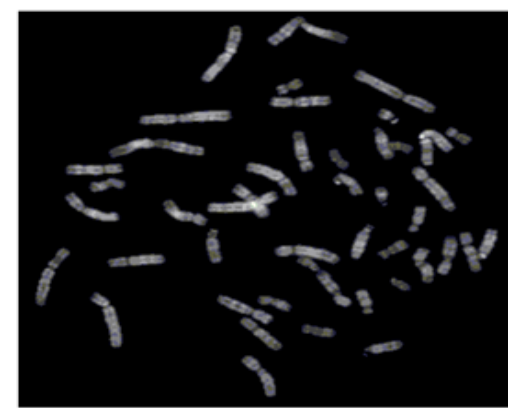

(a)

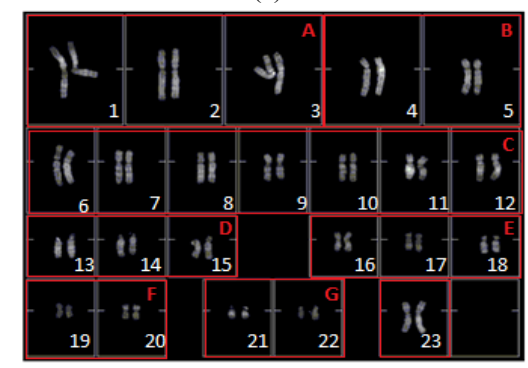

(b)

Figure $1:$ (a) A metaphase plate for a normal female cell; (b) The corresponding respective karyotype.

\section{B. Automated Karyotyping Systems [AKS]}

The idea of using computers to automate the manual process of karyotyping was initially presented in the year of 1964 by Ledley. Casperson et al., in later 1970s, added a major dimension to this idea by proposing the features (size, length, CI, band patterns) for the development of Automated Karyotyping systems [AKS]. This marked an exponential increase in the scientists designing AKS. Such systems were obviously expected to offer countless clinical advantages such as, swift screening of genetic specimens, interactive and graphical environment, faster in the completion of the karyogram, allowing eminence publishing, improved understanding and analysis of the image, decrease labour costs and enable long-term storage [10].

\section{Commercial Software for AKS}

Marketable systems for AKS were introduced as early as 1964 to classify chromosomes. In past few years, most of the commercial AKS have been developed claiming encouraging success rates (60-90\% accuracy) however, do not perform significantly when deployed practically in the genetic labs and fail to successfully karyotype images with touching overlapping and occluded chromosomes. In such cases, the performance is restricted to mere 10-15\% of accuracy [11].

A rudimentary AKS system designed and developed at the Medical Research Council (Scotland) and Lawrence Berkeley Laboratory (California, USA) delivered encouraging performance but suffered from serious limitation of high falsepositive rate of 5.5. The AKS developed by Cancer Research Centre of Pathology laboratory, University of MissouriColumbia faced challenges in acceptance by the cynotgenetists due to inability of the software to segment touching and overlapping chromosomes. VISUSIMAGING developed by
Russian institute addresses this limitation delivering acceptable performance and leading to the extension CYTOVISION System presented by British Applied Imaging Corporation. Integrate d CAD scheme was another noteworthy contribution by Xingwei Wang from University of Oklahoma [12]. Few systems for automated karyotyping are also used as learning aids for the beginners in the genetic labs. Regardless of the momentous investigations and contributions in AKS and in spite of the progress made, genetic and the clinical laboratories still have inhabitations in easy acceptance and practical deployment of fully automated systems, thus still keeping this as an open area of research.

\section{Databases Used in the Devlopment of AKS}

Wide availability of the databases has geared up the ongoing research in the automation of chromosome analysis [12]. Some of the popularly used typical databases (gray images) in the literature are Copenhagen, Edinburgh, Philadelphia, Bioimlab, LK1 and MFISH .Table IIdescribes and compares the image characteristics of three public datasets (Copenhagen, Edinburgh, and Philadelphia) [7].

Recently, a new dataset developed by Enea Poletti (BioImLab) [6] has been made publically available for the research community. This dataset set is composed of huge number of both overlapping and touching chromosomes thus helping the experimentations to prove the efficacy of the algorithms developed by researchers. It also contains segmented individual chromosomes. Artem K. et al. created another chromosome dataset LK1 [13] of relatively low quality chromosomes. This dataset was developed in in association with the IMM, Lisbon (Institute of Molecular Medicine) and has been widely used to test the classification and pairing algorithms. The MFISH chromosome Imaging Database was developed by former Laboratory of Imaging and Video Engineering. The image set contains $200 \mathrm{M}-\mathrm{FISH}$ Images [14].

\section{E. Block Diagram and Basic Steps in AKS}

A typical AKS usually includes four fundamental handing out steps: pre-processing, segmentation, feature extraction and chromosome classification. Figure demonstrates these basic building blocks along with the results of the intermediate processes. Figure (a) depicts original metaphase (BioImlab developed by Enea et al.) Figure 2(b) illustrates the segmentation of metaphase images to obtain individual isolated chromosomes after appropriate image enhancement techniques. Figure 2(c) shows the computation of one such feature, band profile (horizontal bands which are present along the longer axis of the chromosomes) that enable the further classification of the chromosomes. Figure 2(d) shows an example of classifier, ANN used to produce a karyotyped image at the output as seen in 2(e). Some other operations involved in a fully automatic or semi-automated chromosome analysis include identification of isolated, touching and overlapping clusters and disentanglement of the clusters before their classification. 


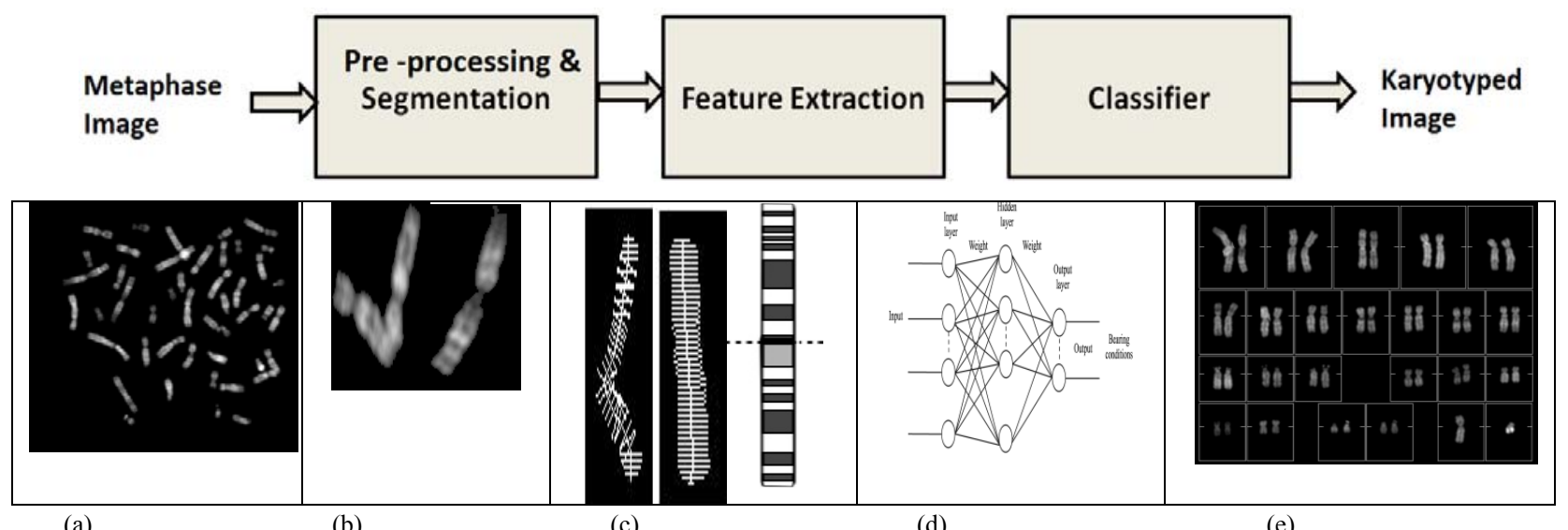

(a) (b)

(c)

(d)

(e)

Figure 2: AKS for classification of banded chromosomes. The components include: (a) Input metaphase image (b) Segmented chromosomes (c) Feature Extraction(d) Classifier (ANN as an example of classifier) (e) Final Karyotyped output.

\section{SURVEY OF IMAGE ENHANCEMENT / PREPROCESSING TECHNIQUES IN AKS}

Image enhancement is the first step in the design and development of AKS. Images captured during the metaphase stage of cell division are prone to various types of noise. Algorithms for image enhancement improve the quality of the images and increase the possibility of extracting better features for improved classification and karyotyping of the chromosomes [15]. Effective algorithms are proposed in the literature for improving signal to noise ratio, for background suppression and increasing the quality of low contrast band features, ultimately leading to effective automated systems for chromosome classification.

A wide assortment of image pre-processing algorithms are explored and examined for improving the metaphase spreads. Smoothening and sharpening filters implemented using Sobel, Roberts, campus operators, Laplacian pyramids have been popularly used and are proved effective $[14,15]$.

A novel technique based on cubic spline wavelet transform was devised by Wu et al. [16], whereas Wang et al. $[15,17]$ applied a family of differential wavelet for enhancement of chromosome images. Both these methods have limitations in terms of being shift and rotation invariant and requires additional memory because of over-complete representation. Seyed et al. [18] proposed another methodology based on plotting histograms and utilizing histogram matching techniques using iterative and adaptive approaches to enhance the contrast ratios of the chromosomes in metaphase images. The results were encouraging and acceptable, however adaptive choice of the executing parameters proved to be a major hindrance considering atomisation of manual karyotyping.

MFISH imaging picked up momentum in genetic lab. Pre-processing operations included operations like back ground correction, feature normalization and color compensation [19]. Due to the non-uniform illumination there are intensity variations which result into non-flat background surfaces. The observed signal at pixel y is modelled using the equation as detailed in eq 2.1

$\mathbf{y}=\mathbf{E}^{*}\left\{\mathbf{C}^{*} \mathbf{x}+\mathbf{b}\right\}+\mathbf{n}$

Where, $\mathrm{x}=6 \mathrm{X} 1$ vector of the true signal, $b=d c$ offset of the CCD and various factors causing background elevation,

$\mathrm{n}=$ Noise of the imaging device such as white and shot noise

$\mathrm{E}=6 \times 6$ diagonal matrix of exposure times,

$\mathrm{C}=6 \times 6$ colour spread matrix,

It is necessary to develop additional image enhancement models that enhance the medical information and explore their utility to assist clinicians in diagnosis and further research. Techniques for removing low frequency shading components, staining debris and microscopic noise will also lead to improvement in the overall performance of AKS and such techniques need to be devised to intensify the overall performance measures of chromosome classification and therefore is an important step in computerized AKS.

\section{SURVEY OF CHROMOSOME SEGMENTATION TECHNIQUES}

Segmentation is the process of separating individual chromosome (object of interest, foreground) from a metaphase spread (background) [19]. All the pixels belonging to sae chromosome or enclosed by the boundary of a particular chromosome are assigned the same label using region growing, region merging or thresholding operations [20]. A metaphase image has either an isolated chromosome or a cluster of touching /overlapping chromosomes. Segmentation and feature extraction of an isolated chromosome is relatively simple when compared to touching, occluded and overlapping chromosomes. The segmentation approaches need to be specifically devised to disentangle the overlapping and touching chromosomes in the metaphase image before the technician proceeds for the extraction of the features [21]. Some of the widely reported successful methods include use of Histogram, edge / region based and combination of various techniques (hybrid) [21].

\section{A. Segmentation of Isolated Chromosomes:}

Similarity based methodologies utilizing global thresholding techniques are widely reported for the segmentation of isolated chromosomes in a metaphase image [22]. The chromosomes of various classes largly differ in the size and length. Due to this watershed based segmentation 
algorithms have limited success for the segmentation of the chromosomes in group $\mathrm{E}$ and $\mathrm{F}$. Presence of noise in low contrast chromosomes make the process further challenging [23]. Grisan et al. [24] presented a space-variant thresholding scheme with very high segmentation accuracies for simple cases. But the algorithm substantially fails when the chromosomes are bent or clusters. A hybrid approach for segmentation was proposed by Nemanje et al. [25]. The method automatically determines background colour, chromosome colour and tolerance values but requires user interaction. This is not favourable when designing an efficient AKS.

Active shape models, active counters have also been explored, however the need further investigation due to concavity of chromosomes [26]. Recently, Enea et al. [27] explored thresholding strategies to report serious limitation imposed by appearance variability and background inhomogeneity. Researchers also explored usefulness of entropy and watershed segmentation and ultimately reported its limited success in cases of artefacts in MFISH images [21].

\section{B. Separation of Touching and Overlapping Chromosomes:}

Automated segmentation techniques for efficient disentanglement of touching and overlapping clusters and blob of chromosomes are the most crucial issue in AKS. This issue has gained most attention of the researchers. Chromosomes are non-rigid structures floating in the nucleoplasm of the nucleus. They therefore exhibit numerous possibilities of touching and overlapping forming clusters of multiple chromosomes. Many researchers have contributed in the development of segmentation algorithms for AKS.

Charters [20] demonstrated a hybrid approach based on trainable shape models and classification indication on synthesized overlaps of $\mathrm{X}$ and $\mathrm{T}$-shape and reported improvement in the performance. Synthetic patterns are considered and the investigation is limited only to two overlaps. The same evidence could in principal be used for larger complex cluster but its analysis is still unexplored. Even two overlaps $\mathrm{X}$ and $\mathrm{T}$ may be practically composed of multiple chromosomes. There still remained outsized scope to consider other types of overlaps like K shape, D shape which will certainly make the system flexible and robust.

Agam et al. [22] and Lerner et al. [28] presented another novel approach comprising of unique banding characteristics of the chromosome to disentangle the cluster. Popescu et al. [29] utilized an effective methodology of analysing the boundary and axis and the approach delivered comparable results. However, in some approaches using genetic algorithms [30] quantitative and qualitative analysis of this approach is yet unexplored from AKS prospective.

Shunren et al. [31] devised smart algorithms exploiting the contour characteristic for chromosome incision. Hybrid approach by Gunter et al. [32] combining rule based system and constrained discriminant analysis provided acceptable results but had limitations in terms of speed and choice of parameters. Similar algorithm devised by Sahar et al.[33] failed in cases where chromosomes touch end to end, which are quite probable in a metaphase image, whereas algorithm presented by Srisang et al. [34] fails considerably to locate the centre of overlapping areas in few cases.

In mid 1990s, MFISH technique was developed. Karvelis et al. [35] and Schwartzkopf et al. [14] have demonstrated maximum likelihood based methods and entropy estimation methods for separation of touching and overlapping cluster. Table I compares the performance of few best methods for separation of chromosome overlaps.

Most of the researchers contributing in the development of algorithms for extrication of overlapping chromosomes have considered single overlaps restricted to only two overlaps. Considering natural variability of shape it is extremely important to devise additional algorithms that increase segmentation accuracies and thus classification accuracies. Addressing this issue and providing an acceptable and encouraging performance in the genetic labs is still a challenging task and needs further investigations from the perspective of practically deployable AKS.

\section{Automated Identification of Single and Overlapping Chromosomes:}

Isolated and overlapping chromosomes demand execution of different segmentation algorithms. Another issue which has received meagre attention is automated identification of an isolated chromosome and a blob of touching/overlapping chromosome in a metaphase spread. Rahimi et al. [37] proposed a neural network based approach to deliver just satisfactory results.

Table I: Performance comparison of the methods reported in the
literature for separation of overlapping chromosome cluster
\begin{tabular}{|c|l|l|l|}
\hline $\begin{array}{c}\text { Reference } \\
\text { No. }\end{array}$ & $\begin{array}{c}\text { Data } \\
\text { Set } \\
\text { (No. of } \\
\text { images) }\end{array}$ & $\begin{array}{c}\text { Data Set } \\
\text { Composition } \\
\text { (no. of chromosome } \\
\text { in the overlap and } \\
\text { type of overlap) }\end{array}$ & $\begin{array}{c}\text { Accuracy } \\
\text { (\%) }\end{array}$ \\
\hline$[20]$ & NA & 2 (T- shaped) & 84.8 \\
\cline { 3 - 4 } & 2 (X-shape) & 92.3 \\
\hline$[22]$ & 25 & 2 & 88 \\
\hline$[23]$ & 162 & 5 & 90 \\
\hline$[28]$ & 46 & $2(1$ overlap) & 82.6 \\
\hline$[29]$ & 219 & NA & 89 \\
\hline$[31]$ & 40 & NA & 92 \\
\hline$[34]$ & 35 & $2(1$ overlap) & 80 \\
\hline$[36]$ & 46 & $2(1$ op) & 94.6 \\
\hline & & $\begin{array}{l}2(1 \text { overlap) } \\
2,3,4(2,3,4\end{array}$ & 100 \\
\hline$[84]$ & 60 & $\begin{array}{l}\text { overlaps }) \\
5,6(5,6 \text { overlaps })\end{array}$ & 85 \\
\hline
\end{tabular}

From the perspective of a fully automated chromosome analysis, it is therefore necessary to identify and distinguish between a chromosome cluster and an isolated chromosome. A procedure that distinguishes between an isolated ('S' shaped) chromosome, touching chromosome (' L' chromosome) and overlapping chromosomes (' $\mathrm{X}$ ' shaped) is necessary before application of the extrication algorithm. This problem has received very less attention in the literature inspite of being an important step in a fully automated chromosome analysis system.

\section{SURVEY OF FEATURES AND FEATURE EXTRACTION APPROACHES FOR AKS}

Features extracted and the algorithms used for extraction of the features play a substantial role in the performance of the developed AKS. A metaphase image 
imposes numerous challenges on the task of karyotyping. Some of these challenges include resolutions of the metaphase image, quality of the image, number of overlapping and touching chromosomes, bend and highly curved chromosomes [38]. Developing efficient algorithm to extract appropriate features irrespective of the challenges imposed demand great research efforts. Next sub-section details the features used in the development of $\mathrm{AKS}$ and the reported algorithms are detailed further.

\section{A. Features used for AKS}

The traditional features used for the computerized karyotyping of the metaphase image are categorized as dimensional, geometrical and pattern based features. Length of the chromosome, the area of the chromosome in terms of the number of pixels is popularly used at a preliminary level to decide the group of the chromosomes. Other morphological features includes perimeter of the chromosomes as well. The category of the geometrical features includes normalized area and centromeric ratio or arm ration. Pattern based systems are of outmost importance when considering the sub-group classification in 23 classes. Each chromosome is categorized with a band pattern which is unique to a particular pair in respective class. Various algorithms are reported to extract the sequence of the band patterns. Polarisation of the chromosomes is also an important consideration when extracting the features of the chromosomes. The extracted sequences of the band will match and help the process of pairing of chromosomes only when the chromosome position match each other from the perspective of the longer and the shorter arm. Literature reports medial axis as a fundamental term to extract the axis of the chromosome and consequently the sequence of light and dark bands in terms of varying intensities. Sweeny et al [39] proposed the extrication of features in frequency domain and other researchers also examined various algorithms based on profile densities [40]. Literature also reports few novel features like Normalized Average Gray Density [NAGD], and mutual information [41], beyond the traditional features used manually by the cytogentist in genetic labs.

\section{B. Feature Extraction and Selection Methods :}

Roshtkhari et al. [42] presented novel algorithms for extrication of features in highly curved chromosomes. Akila et al. [43] proposed a hybrid algorithm but the effectiveness and success is limited to only groups A, B and C. Jau et al. [44] developed an efficient approach for medial axis determination and. Shadab et al. and Lerner Et al. [45] developed an algorithm to estimate a single-line medial axis but algorithm failed in cases of severly bend chromosomes. Only 5 types of chromosomes were however classified as the part of the study. It is necessary to evaluate and confirm the importance of the features treating it as a 24 class problem. .

Seung et al. [47] selected some features: relative length, normalized Density Profile [DP] and Gunter et al. [48, $49,50]$ proposed a novel approach exploiting the dominant points. Severely bent chromosomes are however not considered in this study. Shape variability in chromosomes is very natural due to its non-rigid nature. The efficiency of the algorithm in such cases needs to be further examined. The method formulated by Enea et al. [51] finally lead to good classification accuracies, whereas approach by Wang et al. [52] to detect the centromere position for chromosome polarization has been examined only on limited types of chromosomes. In another approach shape representors have been examined using wavelet [53, 54] and ANN [55] but even these methods have limited success.

Accurate feature vector formed with efficient extraction algorithms may lead to (but not necessarily guarantee) improved classification accuracy and thus enhance the overall performance of $\mathrm{AKS}$, demanding further research in AKS [56].

\section{PAIRING AND CLASSIFICATION OF CHROMOSOMES}

Pairing and the classification of chromosomes, using the extracted features is the last step in AKS. A wide assortment of approaches including neural networks, fuzzy based approaches and artificial intelligent algorithms are explored and reported in the literature. Various fuzzy based approaches [57, 58,59], Expert systems [60], transportation algorithm [61], sub-region search iteration [62] and space prototyping algorithm [63], Hidden Markow Models [64 ,65 ] are experimented for pairing and classification of human chromosomes $[66,67,68]$ considering database of gray and MFISH images.

Among the reported approaches, statistical algorithms and artificial intelligence approaches have delivered appreciable performances. Xingwei et al. [69] proposed a novel two-layer classification approach using ANN, whereas a standard genetic algorithm based approach is proposed by Munot et al. [70] explored unique approach incorporating use of rough set theory for the segmentation of chromosomes and further experimented use of genetic algorithms to achieve appreciable results.

Table II compares the classification accuracies of few methods reported in the literature. It must be acknowledged that this comparison is only being indicative, because of the different datasets used. It gives an over-view about the usability and modularity of the classifiers. MFISH imaging is proved to be extremely useful in cytogenetics, attracting many researchers to contribute in development of AKS. First paper on MFISH technique was published in 1996 by Speicher et al. and further in 1996, he proposed semi-automated image analysis consisting of Segmentation, thresholding and classification stages. These efforts revolutionized chromosome imaging. MFISH Image analysis was fully automated by modelling the task as a 5 feature 24 class pattern recognition problem and using region merging process. Schwartzkopf et al. [71] experimented a segmentation algorithm for MFISH images that minimizes the entropy of classified pixels. The method successfully decomposed the clusters of touching and overlapping chromosomes. But due to its poor computationally complexity, he further exploited maximum likelihood technique. Their approach outperformed the previously reported classification methods. Sampat et al. [72] explored pixel - by pixel classification for non-overlapping chromosomes and achieved $95 \%$ accuracy and further proposed supervised parametric and non -parametric classification of chromosome images. They reported to success of $\mathrm{k}$-nearest neighbor method with $\mathrm{k}=7$ for achieving highest classification accuracy.

Wang et al. [73] presented a novel algorithm that utilizes Fuzzy $\mathrm{C}$ means clustering to achieve improved classification accuracy. Karvelis et al. [74, 75] reported watershed based method for segmenting the chromosomes. A 
region based classifier was implemented to classify the segmented chromosomes. The method achieved overall accuracy is of $82.4 \%$. Choi et al.[ 76, 77] explains joint segmentation of MFISH images to report an overall segmentation accuracy of $98 \%$. Choi et al. [78] explains the algorithm for removal of non-flat background from the MFISH images and reports an improvement in classification accuracy by a factor of 10 after background correction. Petros et al. [79] devised an approach based on Bayes classifier and verified the efficacy of the algorithm using MFISH image database.

Table IIError! No text of specified style in document.: Comparisons of the classification accuracies reported in the literature

\begin{tabular}{|c|c|c|}
\hline $\begin{array}{l}\text { Method- } \\
\text { ologies }\end{array}$ & $\begin{array}{c}\text { Accuracy } \\
\text { (\%) }\end{array}$ & Observations \\
\hline $\begin{array}{c}\text { Dynamic } \\
\text { time warping }\end{array}$ & 81 & Heavy computation load \\
\hline $\begin{array}{c}\text { Hidden } \\
\text { Markow } \\
\text { Model }\end{array}$ & $\leq 97$ & $\begin{array}{c}\text { Dependency on parameter } \\
\text { estimation }\end{array}$ \\
\hline $\begin{array}{l}\text { Artificial } \\
\text { Neural } \\
\text { Network }\end{array}$ & $\begin{array}{c}94 \\
67-97 \\
98\end{array}$ & $\begin{array}{c}\text { Need of optimization } \\
\text { Relatively low accuracy of } \\
\text { group C } \\
\text { Experimented only on group E }\end{array}$ \\
\hline $\begin{array}{c}\text { Genetic } \\
\text { Algorithm }\end{array}$ & $91-95$ & \multirow{2}{*}{$\begin{array}{l}\text { Unable to handle incomplete } \\
\text { cells and bend chromosomes }\end{array}$} \\
\hline $\begin{array}{l}\text { Sub space } \\
\text { prototype }\end{array}$ & $\leq 95$ & \\
\hline Similarity & 90 & Shape dependability \\
\hline
\end{tabular}

The results obtained were superior due to use of vector median filtering. A combination of other sophisticated classifier with other feature extraction algorithms is expected to further improve the results.

MFISH technology has seen major advancements in last few decades leading to significant contributions in the development of highly efficient AKS. However, few challenges in terms of cost, misclassifications and misinterpretations are reported by Lee et al [80]. Another very important limitation which seems to have received comparatively less attention in the literature is the computational complexity of the overall process.

The problem of MFISH image classification is formulated as a five feature 24 class pattern recognition problem. Processing a multispectral set of five images, each one corresponding to a particular dye, increases the overall computational complexity by five times as compared to gray scale imaging. Recently, Hua et al. [89] introduced embedded M-FISH image coding (EMIC) to reduce the issue of memory requirements, transfer and computational cost. Goienetxea et al. [81] explored a pipeline approach for image analysis in AKS.

Building intelligent classifiers with expert knowledge for karyotyping of MFISH and Gray images is major impediment in the development of AKS. A fully automated and highly effective chromosome analysis system which can be directly deployed in clinical environment, made available as commercial package for genetic lab and that is a facilitating tool for doctors still need further investigations and additional experimentations.

\section{DISCUSSION AND CONCLUSION}

Despite the scrupulous efforts to develop a fully automatic chromosome classification system, it has limited success in comparison with the performance and results delivered by an expert in genetic laboratories. One of the possible areas of further expansion for improved performance includes adequate utilization of the expert knowledge and experience to develop decision making ability. Enormous efforts to develop automatic chromosome classification techniques have been made so far. AKS is an attempt to completely circumvent or minimize the human effort in the process of karyotyping. The system must therefore have both, expert knowledge and experience of the trained cytogeneticist from the clinical acceptability prospective. This makes the development of the AKS difficult and challenging and therefore demands additional research. Some of the most important findings, challenges and issues in the development of fully automatic chromosome analysis systems for classification of gray and MFISH images are discussed below:

- MFISH image analysis proved to be a boon in development of AKS, however the complexity of the algorithms demands substantial memory and involve time intricacies that reduces the speed. In the multivariate analysis used in MFISH imaging, a 5-element mean vector is to be multiplied with $5 \mathrm{X} 5$ covariance matrix. Considering computations at pixel level, this leads to 30 multiplications/pixel and 24 additions/pixel and class. Since there are 24 classes and the image size used is 517 X 645 leading to final count of approximately 240 million multiplications/additions for each image. It is necessary to devise and explore the utility of univariate algorithm which addresses the issue of computational complexity and still delivers comparable performance index. This issue needs further investigations and detailed analysis from the perspective of AKS. .

- MFISH Imaging brought revolution in field of AKS and simplified the task of extrication of overlapping chromosomes. Every chromosome of a particular class absorbs a specific dye and color information eases the task of separation of the cluster. But the overlap formed may also involve the chromosomes of the same class. The entire cluster would then be of same color and make the task of separation impossible. So this issue needs to be addressed in MFISH imaging.

- In the recent past, the research community and the genetic laboratories have experienced a paradigm shift as the research is now focused on deploying intelligent and a fully automatic karyotyping system with ideally, absolutely no manual intervention. However, to ensure human-like, acceptable and reliable performance of such systems it is mandatory and indispensible to incorporate a learning and a decision taking capability which is necessarily in consistent and in line with human learning. Similar to cytogenetic experts in genetic laboratories, such intelligent AKS should exhibit decision taking ability and form implication using the knowledge acquired, training phases and the learning from the earlier databases of metaphase image to be karyotyped. Human beings continue to learn throughout their life cycle. The process of learning new information continues without disregarding or forgetting previously learned and acquired information and knowledge. This however, elevates the stability-plasticity dilemma. The popular and extremely widespread use of neural networks as classifiers in AKS 
unfortunately requires the disposal of existing classifier and demand time consuming. Artificial neural network based classifiers lying on the "stability" end of the spectrum, thus suffer from "Catastrophic Forgetting" $[\mathrm{CF}]$, which is the loss of all previously acquired information. Regardless of the huge assortment of the classifiers and hierarchical classifying approaches being analyzed in the development of AKS there still remains an extensive opening to investigate the expediency incremental learning approaches to strike the expected stability-plasticity balance in the classification task. This concern has received very limited attention and has been partially attempted to very few classes for karyotyping $[82,83]$. From the perspective of practical deployment of intelligent AKS with imbibed expert knowledge, it is indispensible to experiment incremental approach for 24 class problem.

- Most of the higher classification accuracies reported in the literature are obtained when the approaches were tested and examined only on few Denver groups or limited types of chromosomes or when only a part of dataset is used. The retention of the same classification accuracies considering the chromosome classification as 24 class problem needs to be examined. Efficiency of the algorithm considering the variations and diversity in the independent data bases must also be examined. Moreover $\mathrm{AKS}$, being an application of use in medical domains needs to have very high classification. There is a genuine need to increase upon the reported classification by developing better system with high classification accuracies.

- Most of the karyotyping systems consider the task of chromosomes classification as a 24 class problem. This will only be applicable to normal cell containing all 46 chromosomes. An abnormal human cell may have more or less number of chromosomes. So similarity based pairing approach and classification methods handling abnormal / incomplete cells (with excess or less count of chromosomes) needs to be further developed and fully experimented before bringing the automated systems to direct practical use in the genetic labs.

- Researchers have carried out extensive research to analyze the features of the chromosomes with an objective of deriving an optimum feature vector for enhancing the classification accuracies. They have reported the most dominant features considering all the subgroups (24 classes) simultaneously, i.e. overall chromosome classification system. But the dominant features may vary for every group. To the best of our knowledge there has been no report on the significant features evaluated for every group ( 7 groups). This study will in particular be useful when two stage chromosome classification approaches is used. Having identified the group of the chromosome only the significant features of that particular group can be further extracted before classifying it in its respective subgroup or subclass.

- Chromosomes are non-rigid in nature and therefore exhibit elevated shape variability. It is very common to have highly curved chromosomes with multiple bending centers. Literature reports algorithms to straighten the chromosome before its feature extraction. But such approaches only consider one bending centre (bend at the centromere) which may not always be the case. Most of the other approaches that derive the centerline of the chromosome fail when the chromosomes are severely bent (very high bending angles). It is necessary to develop efficient feature extraction algorithm capable of handling highly bend chromosomes and are computationally inexpensive.

- Human interventions are reported in many automated systems and are thus interactive. "Manual e-cutting" is required by an expert cytogenetists to guide the automated system towards further classifications. When dealing with huge number (above 200) of samples in Genetics lab, this manual procedure becomes very tedious, needs a trained expert and is time consuming. So it of outmost importance to develop systems with thorough image understanding and with expert knowledge for complete automation.

- The majority of the algorithms and approaches devised to disentangle the cluster of touching and overlapping chromosomes are tested on limited cases such as a single overlap or an overlap of ' $X$ '. An efficient AKS must be able to extricate cluster of chromosomes with numerous overlaps and involving numerous chromosomes [84]. Considering the possibility of any unpredictable overlaps in varying degrees and varying shapes it is extremely important to formulate effective and efficient segmentation and feature extraction algorithms to build intelligent classifiers to function of independent of the count of chromosomes in the overlap and shape of the overlap.

- Automated partition of touching chromosomes is also a major challenge in AKS because it hurdle the feature extraction process. Limited contributed are reported in the literature regarding this issue. It is essential to develop algorithms to separate the chromosomes that touch each other at varying degrees and angles. .

- To develop and deploy a fully operational AKS with absolutely no human interference, it is imperative to imbibe expert knowledge of trained cynotgenetists to automatically categorize and discriminate between the non-overlapping, touching and the overlapping cluster in a metaphase. The overlapping cluster needs to be separated before the application of feature extraction algorithm, whereas features of an isolated chromosome can be directly extracted soon after its segmentation. This important issue of AKS has been given relatively less consideration by the researchers.

- The chromosome needs to be polarized as per ISCN standard before extracting the feature vector. This problem has comparatively received less attention. Most of the approaches assume that the chromosomes are already polarized which may not be applicable from clinical genetic lab perspective. Another issue is identifying the centromere of the chromosomes. Though the task is simple when the chromosomes are vertical, it actually challenging when the chromosomes are bent. It is indispensable to address this problem with some supplementary research.

- In most of the cases when testing the systems for various images, chromosome spreads are photographed through a microscope. Digitally photographed metaphase images are 
captured with microscope. So there is a possibility microscopic noise in the image. Noise due to staining, staining debris, Gaussian, salt and pepper noise interference in the images must be considered. Most of the developed systems are not tested and verified with these considerations. So research based on noise analysis is also required to be carried out. It is necessary to develop additional algorithms that eliminate the effect of staining, sample defects and imaging conditions. This is of outmost importance when applying the AKS in clinical environment. Real and practical database with such defects must also be made available for the research community for further experimentation.

\section{ACKNOWLEDGMENTS}

Author is immensely grateful to my mentor, Dr. Madhuri Joshi for her continuous support and motivation and technical inputs. Author is also deeply thankful to Dr. V. Bairagi and Dr. A. D. Anuse for their perpetual support and important inputs. This research was funded by Department of Science and Technology.

\section{REFERENCES:}

[1] Introduction to medical Genetics HemmaPurandare

[2] http://leavingbio.net/cell\%20division.htm

[3] http://geneticssuite.net/node/24: Karyotype : the nature of eukaryotic nuclear chromosomes

[4] http://www.mendeley.com/catalog/iscn-standardideograms/\#page-1

[5] current protocols in human genetics,http://www.mendeley.com

[6] EneaPoletti, Enrico Grisan, Alfredo Ruggeri, “A modular framework for the automatic classification of chromosomes in Q-band images", Elsevier journal on computer methods and programs in biomedicine, pp 1-11, 2011.

[7] Xingwei Wang, Bin Zheng, Shibo Li, John J. Mulvihill, Marc C. Wood, Hong Liu, "Automated classification of metaphase chromosomes: Optimization of an adaptive computerized scheme", Elsevier Journal of Biomedical Informatics 42, pp 22-31, 2009.

[8] biomed_website_data

[9] Donald C. Graham, T. C. Routley, Gordon T. Dickinson, John 0. Godden, Robert L. Randall, "Identification and Nomenclature of Human Chromosomes", the Canadian Medical Association Journal, vol. 84, pp 1390-1392, June 1961.

[10] Delie Ming, JinwenTian, "Automatic Pattern Extraction and Classification for Chromosome Images", J Infrared MilliTerahz Waves 31, pp 866-877, 2010.

[11] Mousami Munot, Prachi Joshi, Madhuri Joshi, Parag Kulkarni, "Efficient Pairing of Chromosomes in Metaphase Image for Automated Karyotyping", IEEE- EMBS Conference on Biomedical Engineering and Sciences, IECBES - 2012, Langkawi, Malaysia, Dec-2012,pp:916921.

[12] Xingwei Wang, Bin Zheng, Marc Wood, Shibo Li, Wei Chen and Hong Liu, "Development and evaluation of automated systems for detection and classification of banded chromosomes: current status and future perspectives", Journal of Physics D: Applied Physics 38, pp 2536-2542, 2005.

[13] ArtemKhmelinskii, Rodrigo Ventura, and Joao Sanches, “A Novel Metric for Bone Marrow Cells Chromosome Pairing", IEEE Transactions on Biomedical Engineering, vol. 57 , no. 6 , pp 1420-1429, June 2010.
[14] Wade C. Schwartzkopf, Alan C. Bovik, Fellow, and Brian L. Evans, "Maximum-Likelihood Techniques for Joint Segmentation-Classification of Multispectral Chromosome Images", IEEE Transactions on Medical Imaging, vol. 24, no. 12, pp 1593-1610, December 2005.

[15] Yu-Ping Wang, Qiang Wu, Kenneth R. Castleman, and ZixiangXiong, "Image Enhancement Using Multiscale Differential Operators", IEEE International Conference on Acoustics, Speech, and Signal Processing, vol.3, pp 18531856, 2001.

[16] Q. Wu and K. R. Castleman, "Wavelet-Based Enhancement of Human Chromosome Images", International Conference of the IEEE Engineering in Medicine and Biology Society, vol. 20, no 2, pp 963-966, 1998.

[17] Yu-Ping Wang, Qiang Wu, Kenneth R. Castleman, and ZixiangXiong, "Chromosome Image Enhancement Using Multiscale Differential Operators", IEEE Transactions on Medical Imaging, vol. 22, no. 5, pp 685-693, May 2003.

[18] SeyedPooyaEhsani, HojjatSeyedMousavi, Babak.H. Khalaj, "Chromosome Image Contrast Enhancement Using Adaptive, Iterative Histogram Matching", IEEE Iranian Conference on Machine Vision and Image Processing (MVIP), pp 1-5, 2011.

[19] Mousami Munot, Madhuri Joshi, "Towards Automated Karyotyping of Curved Chromosomes", Coimbatore Institute of Information Technology,CiiT International Journal of Artificial Intelligent Systems and Machine Learning, January 2012.

[20] G. Charters, J. Grahman, "Disentangling Chromosome overlaps by combining Trainable Shape Models with Classification evidence", IEEE Transaction on Signal Processing, vol. 50, no. 8, August 2002.

[21] Kostas Haris, Serafim N. Efstratiadis, NicosMaglaveras and Aggelos K. Katsaggelos, "Hybrid Image Segmentation Using Watersheds and Fast Region Merging", IEEE Transactions On Image Processing, vol. 7, no. 12, pp 16841699, December 1998.

[22] GadyAgam, Its'hakDinstein, “Geometric Separation of Partially Overlapping Nonrigid Objects Applied to Automatic Chromosome Classification", IEEE Transactions on Pattern Analysis and Machine Intelligence, vol. 19, no. 11, pp 1212-1222, November 1997.

[23] Enrico Grisan, EneaPoletti, Christopher Tomelleri and Alfredo Ruggeri, "Automatic segmentation of chromosomes in Q-band images", International Conference of the IEEE EMBS CitéInternationale, Lyon, France, pp 5513-5516, August 23-26, 2007.

[24] Enrico Grisan, EneaPoletti, Alfredo Ruggeri, "Automatic Segmentation and Disentangling of Chromosomes in QBand Prometaphase Images", IEEE Transactions on Information Technology in Biomedicine, vol. 13, no. 4, pp 575-581, July 2009.

[25] Nemanja B. Grujic, Nenad V. Ilijic, DraganJankovic, "Hybrid Approach to Image Segmentation Applied on Human Karyotype Determination", IEEE Conference on Serbia and Montenegro, Nis, pp 193-196, September 28 30, 2005.

[26] A. PrabhuBritto and G. Ravindran, "A Review of Deformable Curves from the Perspective of Chromosome Image Segmentation”, Journal of Medical Sciences, 5, pp 363-370, 2005.

[27] Enea Poletti, Francesca Zappelli, Alfredo Ruggeri, Enrico Grisan, "A review of thresholding strategies applied to human chromosome segmentation", Journal of Computer Methods and Programs in Biomedicine, vol. 108(2), pp 679-688, 2012.

[28] Boaz Lerner, "Toward a Completely Automatic NeuralNetwork-Based Human Chromosome Analysis", IEEE Transactions on Systems, Man, and Cybernetics-Part B: 
Cybernetics, Vol. 28, No. 4, pp 544-552, August 1998.

[29] MihailPopescu, Paul Gader, James Keller, Cerry Klein, Joe Stanley, Charles Caldwell, "Automatic karyotyping of metaphase cells with overlapping chromosomes", Elsevier Science of Computers in Biology and Medicine 29, pp 6182, 1999.

[30] Mukul Joshi, Mousami Munot, Madhuri Joshi, Kruti Shah, KetanSoni, "Automated Detection of the Cut-points for the Separation of Overlapping Chromosomes", IEEE- EMBS Conference on Biomedical Engineering and Sciences, IECBES - 2012, Langkawi,Malaysia,Dec-2012, pp:820 825

[31] Xia Shunren, XuWeidong and ShenYutang, "Two Intelligent Algorithms Applied To Automatic Chromosome Incision", IEEE Conference on ASSP, pp 697-700, 2003.

[32] Gunter Ritter, Le Gao, "Automatic segmentation of metaphase cells based on global context and variant analysis",Pattern Recognition 41,pp 38 - 55, 2008.

[33] SaharJahani, S. KamaledinSetarehdan, EmadedinFatemizadeh, "Automatic Identification of Overlapping/Touching Chromosomes in Microscopic images Using Morphological Operators", IEEE Conference on Machine Vision and Image Processing (MVIP), pp 1-4, 2011.

[34] WacharapongSrisang, KrisanadejJaroensutasinee and Mullica Jaroensutasinee, "Segmentation of Overlapping Chromosome Images Using Computational Geometry", Walailak Journal on Science \& Tech; 3(2), pp 181-194, 2006.

[35] Petros S. Karvelis, Dimitrios I. Fotiadis, Ioannis Georgiou and MarikaSyrrou, "A Watershed Based Segmentation Method for Multispectral Chromosome Images Classification", IEEE EMBS Annual International Conference New York City, USA, pp 3009-3012, Aug 30Sept 3, 2006.

[36] Liang Ji, "Fully Automatic Chromosome Segmentation", Wiley-Liss, Inc. Cytometry 17, pp 196-208, 1994.

[37] YaserRahimi, RassoulAmirfattahi, Reza Ghaderi, "Design of a Neural Network Classifier for Separation of Images with One Chromosome from Images with Several Chromosomes", IEEE Conference on Broadband Communications, Information Technology \& Biomedical Applications, pp 186-190, 2008.

[38] Andrew Carothers and Jim Piper, "Computer-aided classification of human chromosomes: a review", Statistics and Computing 4, pp 161-171, 1994.

[39] Nina Sweeneyt, Robert L. Beckert, and Brian Sweeney, "A Comparison of Wavelet and Fourier Descriptors for a Neural Network Chromosome Classifier", International Conference of Engineering in Medicine and Biology Society, vol.3, pp 1359-1362, 1997.

[40] Mehdi Moradi, S. KamaledinSetarehdan, "New features for automatic classification of human chromosomes: A feasibility study", Pattern Recognition Letters 27, pp 1928, 2006.

[41] ArtemKhmelinskii, Rodrigo Ventura and JoãoSanches, "Chromosome Pairing For Karyotyping Purposes Using Mutual Information", 5th IEEE International Symposium on Biomedical Imaging: From Nano to Macro, pp 484-487, 2008.

[42] MehrsanJavanRoshtkhari and SeyedKamaledinSetarehdan, "A novel algorithm for straightening highly curved images of human chromosome", Pattern Recognition Letters 29, pp 1208-1217, 2008.

[43] AkilaSubasingheArachchige, JagathSamarabandu, Joan Knoll, Wahab Khan, Peter Rogan, "An Image Processing Algorithm for Accurate Extraction of the Centerline from Human Metaphase Chromosomes", IEEE International Conference on Image Processing, pp 3613-3616, September 2010.
[44] Jau-hong Kao, Jen-hui Chuang, Tsaipei Wang, "Chromosome classification based on the band profile similarity along approximate medial axis", Pattern Recognition 41, pp 77 - 89, 2008.

[45] Shadab Khan, Joao Sanches and Rodrigo Ventura, "Robust Band Profile Extraction Using Constrained Nonparametric Machine-Learning Technique", IEEE Transactions on Biomedical Engineering, vol. 57, no. 10, pp 2587-2591, October 2010.

[46] Lerner B., Rosenberg B., Levinstein M., Guterman H., Dinstein I. and Romem Y., "Medial Axis Transform Based Features and a Neural Network for Human Chromosome Classification". Elsevier Journal on Pattern Recognition, vol. 28, issue 11, pp 1673-1683, November 1995.

[47] Seung Yun Ryu, Jong Man Cho, SeungHyo Woo, “A Study for the Feature Selection to Identify Giemsa-Stained Human Chromosomes Based on Artificial Neural Network", IEEE International Conference on EMBS, Istanbul, Turkey, pp 691-692, 25-28 October 2001.

[48] Gunter Ritter, GernotSchreib,"Using dominant points and variants for profile extraction from chromosomes", Pattern Recognition 34, pp 923-938, 2001.

[49] Gunter Ritter and GernotSchreib, "Profile and feature extraction from chromosomes", IEEE Conference on Pattern Recognition, vol.2, pp 287-290, 2000.

[50] Gunter Ritter, ChristophPesch, "Polarity-free automatic classi_cation of chromosomes", Elsevier Science of Computational Statistics \& Data Analysis 35, pp 351-372, 2001.

[51] EneaPoletti, Enrico Grisan, and Alfredo Ruggeri, "Automatic classification of chromosomes in Q-band images", IEEE Conference on Engineering in Medicine and Biology Society (EMBS), pp 1911-1914, 2008.

[52] Xingwei Wang, Bin Zheng, Shibo Li, John J. Mulvihill, Hong Liu, "A rule-based computer scheme for centromere identification and polarity assignment of metaphase chromosomes", Elsevier Journal of computer methods and programs in biomedicine 89, pp 33-42, 2008.

[53] L. V. Guimaraes, A. Schuck Jr and A. Elbern, "Chromosome Classification for Karyotype Composing applying Shape Representation on Wavelet Packet Transform", International Conference of the IEEE EMBS, Vol.1, pp 941-943, September 17-21, 2003.

[54] Qiang Wu; Castleman, K.R., "Automated chromosome classification using wavelet-based band pattern descriptors", IEEE Conference on Computer-Based Medical Systems, pp 189-194, 2000.

[55] Jim Piper and Erik Granum, "On Fully Automatic Feature Measurement for Banded Chromosome Classification", Alan R. Liss, Inc. Cytometry 10, pp 242-255, 1989.

[56] Lerner, B., Levinstein, M., Rosenberg, B., Guterman, H., Dinstein, I. and Romem, Y, "Feature Selection and Chromosome Classification Using a Multilayer Perceptron Neural Network", IEEE World Congress on Computational Intelligence., IEEE International Conference on Neural Networks, vol.6, pp 3540-3545, 1994.

[57] Liang Wang, John Yen, "Extracting fuzzy rules for system modelling using a hybrid of genetic algorithms and Kalman filter", Fuzzy Sets and Systems 101, pp 353-362, 1999.

[58] OzySjahputera and James M. Keller, "Evolution of a Fuzzy Rule-Based System for Automatic Chromosome Recognition", IEEE International Fuzzy Systems Conference, pp 129-134, August 22-25, 1999.

[59] Mohammad Ali Akbari and Masayuki Nakajima, "Improving the Automatic Karyotyping Accuracy of the Unrefined Chromosome Features using Fuzzy Logic", IEEE Region 10 Conference on TENCON, vol.3, pp 616619, 2004.

[60] Yuqi Lu, Yili Yan , "An expert system for Banded 
Chromosome Recognition”, IEEE International Conference on Medicine \& Biology Society, Expert System in cytology and histology, 1989

[61] M.K.S. TSO and M.K.S. TSO, "The transportation algorithm chromosome classification", Pattern Recognition Letters 1, pp 489-496, July 1983.

[62] Toru Abe, Chieko Hamada, Tetsuo Kinoshita, "Chromosome Image Recognition with Subregion Search Iteration", IEEE International Conference on Image Processing (ICIP), pp 4169 - 4172, 2009.

[63] QiangWu, Zhongmin Liu, Tiehan Chen, ZixiangXiong, and Kenneth R. Castleman, "Subspace-Based Prototyping and Classification of Chromosome Images", IEEE Transactions On Image Processing, vol. 14, no. 9, pp 1277 1287, September 2005.

[64] John M. Conroy, Tamara G. Kolda, Dianne P. O’Leary, and Timothy J. O'Leary, " Chromosome Identification Using Hidden Markov Models: Comparison with Neural Networks, Singular Value Decomposition, Principal Components Analysis, and Fisher Discriminant Analysis" Laboratory Investigation, vol. 80, no. 11, pp 1629-1641, November 2000.

[65] John M. Conroy, Robert L. Becker, Jr. William Lefkowitz, Kewi L. Christopher, Rawatma1 B. Surana, Timothy J. O'Leary Dianne P. O'Leary, Tamara G Kolda, "Hidden Markov Models for Chromosome Identification", IEEE Conference on Computer-Based Medical Systems, pp 473 477, 2001.

[66] Loris Nanni, “A reliable method for designing an automatic karyotyping system", Elsevier Journal on Neurocomputing 69, pp 1739-1742, 2006.

[67] Mousami Munot, Prachi Joshi, Madhuri Joshi, Parag Kulkarni, "Efficient Pairing of Chromosomes in Metaphase Image for Automated Karyotyping", IEEE- EMBS Conference on Biomedical Engineering and Sciences, IECBES - 2012, Langkawi, Malaysia, Dec-2012,pp:916921.

[68] PraveshBiyani, Xiaolin Wu, and Abhijit Sinha, "Joint Classification and Pairing of Human Chromosomes", IEEE/ACM Transactions on Computational Biology and Bioinformatics, vol.2, no. 2, pp 102-109, April-June 2005.

[69] XinweiFeng, Peisheng Cong, Zhongliang Zhu, Xinyuan $\mathrm{Du}$, "Automated pairing of human chromosomes applying gradient profile and similarity matching algorithm", Elsevier Journal on Chemometrics and Intelligent Laboratory Systems 111, pp 46-52, 2012.

[70] Munot M., Joshi M., Mitra P., "Genetic Algorithm Incorporates with Rough Set Theory: Application to Automated Karyotyping", Indian International conference on artificial intelligence, IICAI 2011.

[71] Schwartzkopf W, Evans B, Bovik A, "Minimum Entropy Segmentation Applied to Multispectral Chromosome Images", Proc. Of $5^{\text {th }}$ IEEE southwest Symposium on Image Analysis and Interpretation, pp 234-237, 2001.

[72] Mehul P. Sampat, K. R. Castleman, A. C. Bovik, "Pixel-byPixel Classification of MFISH Images", IEEE Conference on the Biomedical Engineering Society EMBS/BMES, vol.2, pp 999-1000, 2002.

[73] Yu-Ping Wang, Ashok Kumar Dandpat and Kenneth R. Castleman, "Classification of M-FISH Images Using Fuzzy C-Means Clustering Algorithm and Normalization Approaches", Conference Record of the Thirty-Eighth Asilomar Conference on Signals, Systems and Computers, vol.1, pp 41-44, 2004.

[74] Petros S. Karvelis, Alexandros T. Tzallas, Dimitrios I.
Fotiadis and Ioannis Georgiou, "A Multichannel Watershed-Based Segmentation Method for Multispectral Chromosome Classification", IEEE Transactions on Medical Imaging, vol. 27, no. 5, pp 697-708, May 2008.

[75] P. S. Karvelis, D. I. Fotiadis, I. Georgiou and P. Sakaloglou, "Enhancement of the Classification of Multichannel Chromosome Images Using Support Vector Machines", IEEE Conference on Engineering in Medicine and Biology Society (EMBS), pp 3601-3604, September 2009.

[76] Hyohoon Choi, Kenneth R. Castleman, Alan C. Bovik, "Joint Segmentation and Classification of M-FISH Chromosome Images", International Conference of the IEEE EMBS, San Francisco, CA, USA, pp 1636-1639, September 1-5, 2004.

[77] Hyohoon Choi, Kenneth R. Castlemant, Alan C. Bovik, "Segmentation and Fuzzy-Logic Classification of M-Fish Chromosome Images", IEEE International Conference on Image Processing (ICIP), pp 69-72, 2006.

[78] Hyohoon Choi, Alan C. Bovik, and Kenneth R. Castleman, "Feature Normalization via Expectation Maximization and Unsupervised Nonparametric Classification For M-FISH Chromosome Images", IEEE Transactions On Medical Imaging, vol. 27, no. 8, pp 1107-1119, August 2008.

[79] Petros S. Karvelis, Dimitrios I. Fotiadis, Dimitrios G. Tsalikakis and Ioannis A. Georgiou, "Enhancement of Multichannel Chromosome Classification Using a RegionBased Classifier and Vector Median Filtering", IEEE Transactions on Information Technology in Biomedicine, vol. 13, no. 4, pp 561-570, July 2009.

[80] Charles Lee, David Gisselsson, Charlotte Jin, Ann Nordgren, David O. Ferguson, Elisabeth Blennow, Jonathan A. Fletcher, and Cynthia C. Morton, "Limitations of Chromosome Classification by Multicolor Karyotyping", the American Society of Human Genetics 68, pp 10431047, 2001.

[81] Goienetxea I, Barandiaran I, Jauquicoa C, Maclair G, Grana M, "Image analysis pipeline for automatic karyotyping", Hybrid artificial intelligent systems,LNCS, 7209, pp 392403, 2012.

[82] Prachi Joshi, Mousami Munot, Parag Kulkarni, Madhuri Joshi, "Efficient Karyotyping of Metaphase Chromosomes Using Incremental Learning", The IET Journal of Science Measurement and Technology, Vol.7, Issue 5, September 2013, pp. 287-295.

[83] Mousami Munot, Prachi Joshi, Madhuri Joshi, Parag Kulkarni, "An Incremental Approach for Efficient Karyotyping Systems", Journal of Medical Imaging and Health Informatics, Med. Imaging Health, Inf. 6, Febraury 2016, pp. 221-225.

[84] Mousami Munot, Jayanta Mukhopadhyay, Madhuri Joshi, "A Novel Approach for Efficient Extrication of Overlapping Chromosomes in Automated Karyotyping", Springer Journal of Medical and Biological Engineering and Computing, August 2013, pp. 1-14. 